\title{
Assessment of psychiatric morbidity and quality of life in children and adolescents with cutaneous leishmaniasis and their parents
}

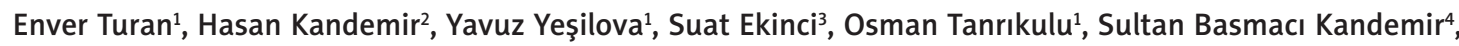 \\ Mehmet Salih Gurel ${ }^{5}$ \\ ${ }^{1}$ Department of Dermatology, Faculty of Medicine, Harran University, Sanliurfa, Turkey \\ ${ }^{2}$ Department of Child and Adolescent Psychiatry, Harran University School of Medicine, Sanliurfa, Turkey \\ ${ }^{3}$ Psychiatry Clinic, Balikli Rum Private Hospital, İstanbul, Turkey \\ ${ }^{4}$ Psychiatry Clinic, Balikli Gol State Hospital, Sanliurfa, Turkey \\ ${ }^{5}$ Department of Dermatology, Istanbul Education and Research Hospital, Istanbul, Turkey
}

Postep Derm Alergol 2015; XXXII (5): 344-348

DOI: $10.5114 /$ pdia.2015.54744

\begin{abstract}
Introduction: Cutaneous leishmaniasis $(\mathrm{CL})$ is a common parasitic infectious disease caused by different species of the leishmania genus. The skin lesions are usually found on exposed areas, especially the face, arms and legs. Although the disease does not cause significant morbidity, the lesions can be troublesome and unsightly. The disease have negative impacts in areas such as patients' psychological well-being, social life and daily activities in adults.

Aim: To determine the frequency of psychiatric morbidity in children and adolescents who have cutaneous leishmaniasis $(\mathrm{CL})$ and to determine the effect of $\mathrm{CL}$ on their levels of depression and anxiety and on their quality of life (QoL). Material and methods: Fifty-four patients with CL (29 males and 25 females), who were 7 to 18 years of age, were assessed with the Child Depression Inventory (CDI) and the State-Trait Anxiety Inventories for Children (STAIC). The patients and their mothers were assessed with the Pediatric Quality of Life Inventory Parent and Child Versions (PedQL-P and C, respectively). This questionnaires were filled in by the control group consisting of 40 healthy children and adolescents (20 males and 20 females) and their parents from the local community matched for age, gender, and education level of the parents.

Results: Both the patient group and the control group had high scores on the depression measurement scale $(t=5.36, p<0.05)$. These measurements also show significant differences between children and adolescents, who were defined as 12 years of age and under as well as older than 12 years, respectively (12 years of age and under $(t=3.14, p=0.04)$; over 12 years $(t=5.37, p<0.001)$ ). However, there was no significant difference between the anxiety scores of the general patient group and the control group when classified according to age. The anxiety sensitivity index scores did not differ in either group from those of the control group. The patients' and the mothers' QoL scores for all of the scales, including all subscale scores, were significantly different from those of the control group (both 12 years of age and under as well as older than 12 years).

Conclusions: The results have shown that the frequency of depressive symptoms is much higher in patients who have $\mathrm{CL}$ than in healthy controls. In addition, the QoL of children and adolescents with $\mathrm{CL}$ and of their mothers was found to be much lower than that of the control group. Therefore, the follow-up for patients with CL who are referred to dermatology clinics should include a psychiatric evaluation. If necessary, they should be referred for psychiatric support.
\end{abstract}

Key words: depressive symptom, anxiety, quality of life, cutaneous leishmaniasis.

\section{Introduction}

Cutaneous leishmaniasis $(\mathrm{CL})$ is a parasitic infectious disease that causes varied spectrum clinical manifestations ranging from small cutaneous papules to gross mucosal tissue destruction. Cutaneous leishmaniasis is distributed worldwide, especially in tropical and subtropical areas where it is caused by a single-celled parasite that is transmitted by sand fly bites [1]. To date, more than

Address for correspondence: Assist. Prof. Enver Turan, Department of Dermatology, Faculty of Medicine, Harran University, 63300 Sanliurfa, Turkey, fax: +90 5053323929, e-mail: enverturan@gmail.com Received: 10.11.2014, accepted: 11.01.2015. 
20 species of leishmania have been described as causative agents of human leishmaniasis [2]. If left untreated, CL can cause chronic ulcerative lesions on exposed areas of the body, including the face, neck, hands, arms and lower legs. The disease in itself does not cause mortality or significant morbidity, although the ugly appearance of the lesions can be troublesome for patients. Because CL causes disfiguring scars on exposed areas and can leave permanent marks, it can lead to social problems, psychiatric symptoms and a lower quality of life (QoL) [3-6].

Several studies have been conducted on the relationship between $C L$ and the psychiatric morbidity and QoL among adolescents and adults who have the disease. However, the psychiatric morbidity and QoL of children with $C L$ together with adolescents who have $C L$ has not previously been examined.

\section{Aim}

In this study, we compare the levels of depression, anxiety and QoL among children and adolescents who have $C L$ to the levels in healthy control subjects. We have used an interdisciplinary approach involving psychiatrists, dermatologists and psychologists to measure the levels of depression, anxiety and QoL in patients with CL.

\section{Material and methods}

\section{Patients}

The study included $54 \mathrm{CL}$ patients (29 males and 25 females) who agreed to participate and gave their informed consent. The control group of healthy children and their parents was formed with the assistance of the Department of Pediatrics of the Harran University. 40 healthy children and adolescents (20 males and 20 females) and their parents admitted to the department of pediatrics for their vaccines, a standard check-up for a sports activity or accompanying their siblings during their clinic visits were included in the study. They also gave their informed consent to participate in the study. Both the study and control groups were divided into two age groups, children (7-12 years, $n=26$ and $n=20$, respectively) and adolescent (13-18 years, $n=28$ and $n=20$, respectively), to consider the effects of puberty on psychological functioning. The period of the study was from 1 May 2011 to 30 April 2013. All of the CL patients who had active lesions were from to the Leishmania Diagnostic and Treatment Centre in the Sanliurfa health directorate and the outpatient clinic at the Harran University Medical School's dermatology department.

The inclusion criteria for the patient and control groups were that they were 7 -to-18 years of age, had had no medical treatment within the previous 3 months, had had no other skin or systemic medical diseases over the same time period and did not have any apparent disabilities.
The study protocol followed the Declaration of Helsinki, all subjects were informed about the study protocol and written consent was obtained from all participants or their guardians. The study was approved by the local Clinical Research Ethics Committee.

\section{Psychological measures}

The Child Depression Inventory (CDI) was used to measure psychological depressive symptoms that included vegetative functions, hedonic capacity, disturbed mood, self-evaluation and interpersonal behaviors. The State-Trait Anxiety Inventories for Children (STAIC) was also used, including two subscales: state and trait anxiety. State anxiety points out the anxiety that is experienced under certain situations, at a certain time; this will change depending on external factors. By contrast, trait anxiety points out to the general feelings of the individual and mirrors the individual's overall tendency to anxiety.

The Pediatric Quality of Life Inventory Parent and Child Versions (PedQL-P and C, respectively) were completed by the participants to show the children's healthrelated quality of life (HRQoL). This scale, which has an overall score and two subscale scores measuring psychosocial and physical health, does not relate only to skin diseases but has been used for children with many different disorders. This scale includes both a parent and a child version for physical as well as psychosocial functioning. A higher total PedQL score indicates better HRQoL. The PedQL-P scale was completed by the mothers. If a mother was not able to complete the questionnaire, or the mother was absent, other caregivers were asked to do so.

\section{Statistical analysis}

SPSS Version 17.0 for Windows (Chicago, IL, U.S.A.) was used to calculate all analyses. Data met the criteria for parametric analysis. Descriptive statistics of the participants' demographic characteristics and clinical features were also calculated. Independent sample $T$ and $\chi^{2}$ tests were used to determine whether there were any differences between members of the patient and control groups in terms of depression, anxiety and HRQoL.

\section{Results}

The patient group consisted of 29 male and 25 female CL patients. Their ages ranged from 7 to 18 years (mean: $12.03 \pm 3.17$ years). The age range of the control group was also 7 to 18 years, with 20 male and 20 female volunteers, all of whom were healthy (mean: $11.50 \pm 2.38$ years). No significant differences were found between the patients and controls for age $(p=0.46)$, gender $(p=0.43)$ and level of education $(p=0.68)$; all statistical analyses were corrected for these factors. 
Of the CL lesions among the patients, $64.8 \%$ were located on the face, $35.2 \%$ on the extremities, $7.4 \%$ on the trunk and $23.4 \%$ in multiple locations (including the face, extremities and trunk); $18.5 \%$ of the patients had two or more lesions were located in different areas of the body. The number of lesions ranged from 1 to 7 (mean: 1.72). The most frequent manifestation was papulonodular lesions (57.4\%), followed by nodulo-ulcerative lesions (27.8\%), plaque (13.0\%) and verrucous (1.8\%). Over half of the patients, $51.8 \%$, presented with single lesions, $16.7 \%$ had two lesions and three or more lesions were observed in only $31.5 \%$ of the patients (Table 1).

The duration of the lesions ranged from 2 months to 14 months, and the mean duration was 3.5 months. The size of the lesions ranged from $0.5 \mathrm{~cm}$ to $9 \mathrm{~cm}$.

For the psychiatric evaluation, both patient and control groups were divided into groups of 12 years of age and under, and older than 12 years in order to look at the psychosocial effects of adolescence. In the patient group, depression scores were higher in the whole group as well as in both age groups than the control group. The scores

Table 1. Clinical features of CL cases

\begin{tabular}{|c|c|c|c|}
\hline \multirow[t]{2}{*}{ Clinical feature } & & \multicolumn{2}{|c|}{ Number of cases } \\
\hline & & Number & Percentage \\
\hline \multirow[t]{3}{*}{ Gender } & Male & 29 & 53.7 \\
\hline & Female & 25 & 46.3 \\
\hline & Total & 54 & 100.0 \\
\hline \multirow{3}{*}{$\begin{array}{l}\text { Number } \\
\text { of lesions }\end{array}$} & 1 & 28 & 51.8 \\
\hline & 2 & 9 & 16.7 \\
\hline & 3 or more & 17 & 31.5 \\
\hline \multirow[t]{4}{*}{ Location } & Face & 35 & 64.8 \\
\hline & Extremities & 19 & 35.2 \\
\hline & Trunk & 4 & 7.4 \\
\hline & Multiple locations & 10 & 18.5 \\
\hline \multirow[t]{4}{*}{ Lesion features } & Papulonodular & 31 & 57.4 \\
\hline & Nodulo-ulcerative & 15 & 27.8 \\
\hline & Plaque & 7 & 13.0 \\
\hline & Verrucous & 1 & 1.8 \\
\hline \multirow[t]{3}{*}{ Complaint } & Asymptomatic & 43 & 79.6 \\
\hline & Itching & 8 & 14.8 \\
\hline & Pain & 3 & 5.6 \\
\hline \multirow[t]{2}{*}{ Place of residence } & Urban & 35 & 64.8 \\
\hline & Rural & 19 & 35.2 \\
\hline
\end{tabular}

in the overall patient group $(t=5.36, p<0.05)$ and, both in the 12 years of age and under $(t=3.14, p=0.04)$ and the over 12 years of age $(t=5.37, p<0.001)$ groups were higher than in the control group. However, in terms of anxiety scores, no significant difference was found in the entire group as well as in their classifications by the age group (Table 2). The QoL scores of both the children and the mothers for all of the scales, including all subscale scores, were significantly lower than those of the control group (both 12 years of age and under, and over 12 years). The patient group's anxiety sensitivity index scores did not differ from those of the control group, in both of the age groups.

\section{Discussion}

Numerous studies have demonstrated that psychiatric disorders resulting from skin diseases may cause lower QoL [7-10]. Any spots or roughness on the skin can deeply affect the morale and self-esteem of individuals as well as their social relationships. The seriousness of these psychosocial outcomes is directly related to how visible the dermatological diseases are. This fact becomes even more striking when the lesions are in cosmetically important areas such as the face [11]. The most common psychiatric morbidities were depression [12, 13], anxiety [8], decreased self-esteem [12, 14], poor body image satisfaction $[12,13]$ and suicidal ideation $[15,16]$.

Cutaneous leishmaniasis is caused by the protozoan parasites that belong to the genus Leishmania and is spread by bites of the sand fly species. The disease can be limited to a single part of the skin (localized cutaneous leishmaniasis) or may involve a broad spectrum of clinical features that will result in common skin lesions (diffuse cutaneous leishmaniasis) [17, 18].

Cutaneous leishmaniasis occurs particularly on exposed areas of the body and produces destructive and disfiguring lesions in these areas [19]. When important areas such as the face are affected, the chronic nature and the scars that can remain even after healing if effective treatment is not given can be demoralizing for patients. Lesions that are visible on the outside can cause the individual to feel stigmatized and can lead to psychosocial problems [3].

Only a few studies have investigated the psychiatric symptoms and QoL among children with dermatological diseases. Two separate studies that looked at the psychiatric symptoms and QoL in children and adolescents who had psoriasis and vitiligo found that disease negatively impacted the QoL of pediatric patients with psoriasis [20]. Also, the psychosocial and total scores of QoL of these children's parents were found to be lower than those of the control group. Although vitiligo was found to be associated with the severity of depression in children with this disease, a similar effect was not demonstrated among adolescents [21]. 
Table 2. Comparison of children and adolescents with cutaneous leishmaniasis and age-matched controls

\begin{tabular}{|c|c|c|c|c|c|c|}
\hline \multirow[t]{2}{*}{ Variable } & \multicolumn{3}{|c|}{$7-12$} & \multicolumn{3}{|c|}{$13-18$} \\
\hline & Patients $(n=26)$ & Controls $(n=20)$ & Value of $p$ & Patients $(n=28)$ & Controls $(n=20)$ & Value of $p$ \\
\hline CDI & $9.72 \pm 6.11$ & $4.5 \pm 3.83$ & 0.001 & $14.25 \pm 4.76$ & $4.50 \pm 2.46$ & $<0.001$ \\
\hline \multicolumn{7}{|l|}{ STAI-C: } \\
\hline State Anxiety & $47.11 \pm 4.04$ & $46.35 \pm 6.05$ & 0.883 & $45.91 \pm 6.58$ & $50.10 \pm 3.66$ & 0.143 \\
\hline Trait Anxiety & $33.94 \pm 8.54$ & $30.45 \pm 7.27$ & 0.207 & $36.41 \pm 40.50$ & $33.90 \pm 10.87$ & 0.143 \\
\hline PedQL-C physical-health & $80.55 \pm 15.40$ & $94.99 \pm 5.00$ & 0.001 & $75.77 \pm 16.05$ & $95.31 \pm 6.29$ & 0.004 \\
\hline PedQL-C psychosocial & $81.94 \pm 12.55$ & $90.83 \pm 6.54$ & 0.011 & $74.51 \pm 13.99$ & $86.33 \pm 7.76$ & 0.027 \\
\hline PedQL-C total & $81.31 \pm 11.39$ & $91.83 \pm 4.76$ & 0.001 & $74.99 \pm 13.95$ & $80.34 \pm 4.74$ & 0.014 \\
\hline PedQL-P physical-health & $79.16 \pm 16.00$ & $96.09 \pm 5.05$ & $<0.001$ & $76.04 \pm 17.54$ & $93.12 \pm 7.03$ & 0.014 \\
\hline PedQL-P psychosocial & $80.20 \pm 9.70$ & $89.7 \pm 8.31$ & 0.005 & $81.66 \pm 6.43$ & $87.83 \pm 5.95$ & 0.043 \\
\hline PedQL-P total & $79.66 \pm 10.59$ & $91.95 \pm 5.80$ & $<0.001$ & $79.20 \pm 874$ & $90.56 \pm 5.12$ & 0.012 \\
\hline Anxiety sensitivity index & $33.45 \pm 5.36$ & $30.1 \pm 5.37$ & 0.310 & $31.83 \pm 4.30$ & $35.40 \pm 6.05$ & 0.112 \\
\hline
\end{tabular}

CDI - Child Depression Inventory, STAI-C - State-Trait Anxiety Inventory for Children, PedQL-C - Pediatric Quality of Life Inventory Child Version, PedQL-P - Pediatric Quality of Life Inventory Parent Version.

The first study of the psychological effects of $C L$ was conducted by Yanik et al. [3]. In this study, the scores for depression and anxiety among adolescent and adult patients with $C L$ were found to be higher than in the control group [3]. This study also found that all groups, but most particularly patients with active lesions, had a significant decrease in QoL. However, the study did not examine the psychiatric effects of $\mathrm{CL}$ on pediatric patients.

Our study found depression scores in children and adolescents with $\mathrm{CL}$ to be significantly higher than in the control group. However, there was no significant difference in the anxiety scores of the two groups. In this study, we also evaluated the patients' families, and the depression scores of mothers of patients with CL were higher than those of the control group. In addition, the study found the psychosocial, physical and total scale scores of both parents and children to be significantly lower than those of the control group in terms of the QoL scales. In our study, patients with high CDI scores and high STAIC-1 and STAIC-2 anxiety scores underwent psychiatric interview. In these patients, the use of antidepressant and anxiolytic medications may increase compliance with treatment [22].

There are several limitations to this study. The working group is a small patient group consisting of only 30 and the patients are all from the same region and from a similar culture. Therefore, further studies using a larger sample size and more diverse populations are required to better identify the relationship between $\mathrm{CL}$ and the psychological status of children and adolescents with the disease. The fact that the patients were evaluated while they were being treated for active lesions in a tertiary health institution is another limitation. This study also excluded patients who had lesions that had healed but left scars.

Data from the literature have shown that $51 \%$ of patients with dermatological problems require psychotherapeutic treatment, $28 \%$ have been willing to have psychotherapy and $38 \%$ of them have received psychiatric help. Liaison services do provide assistance for patients who have psychiatric problems that relate to skin disorders [23]. In a survey study, 23.2\% of medical managers provide psychosomatic treatment in addition to dermatological care and treatment. Patients with CL go mainly to the dermatology clinics where they receive dermatological treatments and either delayed referrals to psychiatric clinics or had no referrals at all [24].

Because chronic skin diseases are not life threatening, the psychiatric morbidity associated with them is neglected, and this can cause severe psychosocial disturbances [25]. Although CL does not result in serious morbidity and mortality, it does affect the patient's physical, mental and social well-being. Patients may experience psychosocial effects during the period of the disease when lesions are active or if ugly scars remain after the spontaneous recovery, and this may reduce their QoL [3]. Recognizing the psychological symptoms of skin disease and addressing them is important in order to improve QoL and ensure a better response to treatment.

\section{Conclusions}

These findings have clearly shown the importance of measuring the levels of depression, anxiety and QoL in children and adolescents who have CL, by using CDI, 
STAIC and PedQL-P and C as screening instruments. The effect of the disease on QoL and on the levels of depression and anxiety should be measured in patients with $\mathrm{CL}$; they need to be examined through interdisciplinary scrutiny that combines dermatological and psychiatric evaluations.

\section{Conflict of interest}

The authors declare no conflict of interest.

\section{References}

1. Dutari LC, Loaiza JR. American cutaneous leishmaniasis in Panama: a historical review of entomological studies on anthropophilic Lutzomyias and fly species. Parasit Vectors 2014; 7: 218

2. Layegh P, Moghiman T, Ahmadian Hoseini SA. Children and cutaneous leishmaniasis: a clinical report and review. J Infect Dev Ctries 2013; 7: 614-7.

3. Yanik M, Gurel MS, Simsek Z, Kati M. The psychological impact of cutaneous leishmaniasis. Clin Exp Dermatol 2004; 29: 464-7.

4. Ranawaka RR, Weerakoon HS, de Silva SH. The quality of life of Sri Lankan patients with cutaneous leishmaniasis. Mymensingh Med J 2014; 23: 345-51.

5. Vares B, Mohseni M, Heshmatkhah A, et al. Quality of life in patients with cutaneous leishmaniasis. Arch Iran Med 2013; 16: 474-7.

6. Toledo AC Jr, da Silva RE, Carmo RF, et al. Assessment of the quality of life of patients with cutaneous leishmaniasis in Belo Horizonte, Brazil, 2009-2010. A pilot study. Trans R Soc Trop Med Hyg 2013; 107: 335-6.

7. Finlay AY, Ryan TJ. Disability and handicap in dermatology. Int J Dermatol 1996; 35: 305-11.

8. van der Meeren HL, van der Schaar WW, van den Hurk CM. The psychological impact of severe acne. Cutis 1985; 36: 84-6.

9. Lewis-Jones S. The psychological impact of skin disease. Nurs Times 2000; 96 (27 Suppl.): 2-4.

10. Jayaprakasam A, Darvay A, Osborne G, McGibbon D. Comparison of assessments of severity and quality of life in cutaneous disease. Clin Exp Dermatol 2002; 27: 306-8.

11. Cunningham SJ. The psychology of facial appearance. Dent Update 1999; 26: 438-43.

12. Koo J. The psychosocial impact of acne: patients' perceptions. J Am Acad Dermatol 1995; 32: S26-30.

13. Devrimci-Ozguven H, Kundakci TN, Kumbasar H, Boyvat A. The depression, anxiety, life satisfaction and affective expression levels in psoriasis patients. J Eur Acad Dermatol Venereol 2000; 14: 267-71.

14. Kent G, Al'Abadie M. Psychologic effects of vitiligo: a critical incident analysis. J Am Acad Dermatol 1996; 35: 895-8.

15. Gupta MA, Gupta AK. Depression and suicidal ideation in dermatology patients with acne, alopecia areata, atopic dermatitis and psoriasis. Br J Dermatol 1998; 139: 846-50.

16. Cotterill JA, Cunliffe WJ. Suicide in dermatological patients. Br J Dermatol 1997; 137: 246-50.

17. Gurel MS, Ulukanligil M, Ozbilge H. Cutaneous leishmaniasis in Sanliurfa: epidemiologic and clinical features of the last four years (1997-2000). Int I Dermatol 2002; 41: 32-7.

18. Uzun S, Durdu M, Culha G, et al. Clinical features, epidemiology, and efficacy and safety of intralesional antimony treatment of cutaneous leishmaniasis: recent experience in Turkey. J Parasitol 2004; 90: 853-9.

19. Sousa AO, Pompeu MM, Solon FR, et al. Disseminated cutaneous leishmaniasis: a patient with 749 lesions. Braz I Infect Dis 2006; 10: 230.

20. Bilgic A, Bilgic O, Akis HK, et al. Psychiatric symptoms and health-related quality of life in children and adolescents with psoriasis. Pediatr Dermatol 2010; 27: 614-7.

21. Bilgic O, Bilgic A, Akis HK, et al. Depression, anxiety and health-related quality of life in children and adolescents with vitiligo. Clin Exp Dermatol 2011; 36: 360-5.

22. Alpsoy E, Ozcan E, Cetin L, et al. Is the efficacy of topical corticosteroid therapy for psoriasis vulgaris enhanced by concurrent moclobemide therapy? A double-blind, placebocontrolled study. J Am Acad Dermatol 1998; 38: 197-200.

23. Fritzsche K, Ott J, Zschocke I, et al. Psychosomatic liaison service in dermatology. Need for psychotherapeutic interventions and their realization. Dermatology 2001; 203: 27-31.

24. Gieler U, Niemeier V, Kupfer J, et al. Psychosomatic dermatology in Germany: a survey of 69 dermatologic clinics. Hautarzt 2001; 52: 104-10.

25. Picardi A, Abeni D, Melchi CF, et al. Psychiatric morbidity in dermatological outpatients: an issue to be recognized. $\mathrm{Br}$ J Dermatol 2000; 143: 983-91. 